\title{
Prevalence of Refampcin Mono Resistant Mycobacterium Tuberculosis among Suspected Cases Attending at Yirgalem Hospital
}

\author{
Mesfin Worku Hordofa ${ }^{1, *}$, Teshome Befikadu Adela ${ }^{2}$ \\ ${ }^{1}$ Hawassa University, chollege of Medicine and Health Scences, Department of Medical Laboratory Science, Hawassa, Ethiopia \\ ${ }^{2}$ Yirgalem Hospital, Yirgalem, Ethiopia
}

Email address:

mesfinwh@gmail.com, mesworku@yahoo.com (M. W. Hordofa), Teshiye57@yahoo.com (T. B. Adela)

\section{To cite this article:}

Mesfin Worku Hordofa, Teshome Befikadu Adela. Prevalence of Refampcin Mono Resistant Mycobacterium Tuberculosis Among Suspected Cases Attending at Yirgalem Hospital. Clinical Medicine Research. Vol. 4, No. 3, 2015, pp. 75-78. doi: 10.11648/j.cmr.20150403.13

\begin{abstract}
Introduction: Tuberculosis (TB) is a chronic communicable disease caused by Mycobacterium tuberculosis (MTB). M. tuberculosis is a slow- growing bacterium, resistant to most conventional antimicrobial agents partly due to its impermeable cell wall. It may persist in a dormant or latent form, unsusceptible to agents targeting growing bacteria. The Gen Xpert MTB/RIF is an automated molecular test which detects DNA sequences specific for MTB and RIF resistance by polymerase chain reaction with fully integrated sample processing in patients suspected of drug sensitive or multidrug resistant pulmonary tuberculosis. Objective: to determine Rifampicin Mono-Resistance Mycobacterium tuberculosis among patient attending atYirgalem Hospital from August-December, 2014.Method:A cross sectional study was conducted on Rifampicin Mono-Resistance in Mycobacterium tuberculosis among patient attending at Yirgalem Hospital from August-December, 2014,Yirgalem, Ethiopia. Result: A total of 236 participants were included under this study. Among these, males (57.6\%) are slightly dominating female. Concerning to treatment history, $177(75.0 \%)$ are new and the rest $59(25.0 \%)$ retreated. Fifty eight $(24.6 \%)$ of the total subjects were suspected for MDR tuberculosis. Twenty two $(9.3 \%)$ of the subjects were smear positive. The highest positive finding of rifampcin susceptible Mycobacterium tuberculosis bacilli observed within age group of 1630 .The overall prevalence of pulmonary tuberculosis was $16.5 \%$. From this prevalence, $3.4 \%$ was shared by Rifampcin monoresistant Tuberculosis. Conclusion: Based on our study finding, The overall prevalence of pulmonary tuberculosis was $16.5 \%$. From these, 3.4\% was account for Rifampicin Mono-Resistance Mycobacterium tuberculosis among study subject. Most of the affected study subjects were productive age group. Therefore, we recommend that there should be enhanced efforts in detection of MDR tuberculosis in study area to control dissemination of the disease among the community.
\end{abstract}

Keywords: Tuberculosis, Rifampcin, Yirgalem Hospital

\section{Introduction}

Tuberculosis (TB) is a chronic communicable disease caused by Mycobacterium tuberculosis (MTB).M. tuberculosis is a slow- growing bacterium, resistant to most conventional antimicrobial agents partly due to its impermeable cell wall. It may persist in a dormant or latent form, unsusceptible to agents targeting growing bacteria $[1$, 2].

The bacterium infects almost one-third of population globally and during the past decade there has been a resurgence of tuberculosis [3].The burden of TB epidemic in developing countries is very high. An estimation of $95 \%$ of TB cases and $98 \%$ of TB deaths occurs in the third world countries, where peoples are especially susceptible to TB because of poor living conditions and limited access to treatment [4].

Based on the World Health Organization (WHO) Annual Global TB Report (2009), Ethiopia ranked seventh in the world for TB burden, with an estimated annual TB incidence (all forms) of 378 new cases per 100,000 persons and 163 new smear positive cases per 100,000 persons per year [5]. The Ethiopian Federal Ministry of Health (FMOH) hospital statistics data has shown that tuberculosis is the leading cause of morbidity, the third cause of hospital admission (after deliveries and malaria), and the second cause of death in 
Ethiopia, after malaria [6]. Based on WHO report in Ethiopia, the incidence of TB of all forms and smear positive TB stand at 341 and 152 per 100,000 population, respectively [7].The prevalence and mortality of tuberculosis of all forms is estimated to be 546 and 73 per 100,000 population respectively [3].

In the Southern Ethiopia Regional State (SNNPRS), TB is among the leading causes for sickness and death (8). As in many other resource-constrained settings, treatment outcomes for tuberculosis have not been satisfactory, mainly due to poor treatment compliance and low coverage of short course chemotherapy (SCC) (9). Delays in the diagnosis and treatment initiation, the devastating HIV/AIDS epidemic and the potential threat of anti-tuberculosis drug resistance represent serious threats to the TB control effort in the region [10].

Rifampicin resistant heralds higher rates of treatment failure and death for the patient and a poor outcome if the isolate is also resistant to izoniazid. Efficacy of rifampicin chemotherapy can be markedly reduced when infections are caused by $\mathrm{M}$. tuberculosis strains that are rifampin resistant [11].

The GenXpert MTB/RIF is an automated molecular test which detects DNA sequences specific for MTB and RIF resistance by polymerase chain reaction with fully integrated sample processing in patients suspected of drug sensitive or multidrug resistant pulmonary tuberculosis [11].

Therefore, to our knowledge there is no previous study on prevalence of Refampcin mono resistant Mycobacterium tuberculosis among suspected cases in the study area.so that the objective of the this study is to determine Rifampcin mono resistant Mycobacterium tuberculosis among patient attending Yirgalem Hospital.

\section{Methodology}

\subsection{Study Setting}

Yirgalem Hospital is a tertiary health care hospital serving the population of south nations and nationalities (SNNPR). There are about 15 million populations are getting service by the hospital from South Nation and Nationalities Region (SNNPR) and the neighbor Oromia region. The hospital located $317 \mathrm{~km}$ south from capital Addis Ababa and $45 \mathrm{~km}$ from Hawassa town. In the hospital a Directly Observed Therapy; Short-Course (DOTS) clinic is operating under the National Tuberculosis and Leprosy Control Program (NTLCP) of Ethiopia, under which the diagnosis of pulmonary TB is followed by examination of three sputum smears (spot morning spot) by Zihel -Nielsen staining method for acid fast bacilli (AFB). Chest radiographs and pathological investigations are also used to support the diagnosis. Patients diagnosed with tuberculosis are referred to the DOTS clinic where they are registered and treated according to the national TLCP guideline (Ministry of Health of Ethiopia (MOH) [11]. In addition hospital also equipped with Gene Xpert that used to determine Refampcin mono resistant Mycobacterium tuberculosis.

\subsection{Study Design}

A cross sectional study was conducted on Rifampicin Mono-Resistance in Mycobacterium tuberculosisfrom August-December, 2014 at Yirgalem Hospital.

\subsection{Data Collection}

Data collection was carried out using structured questionnaire to ask different variables and Laboratory examination on sputum specimen collected for direct microscopy to identify Acid fast bacili (AFB) and determination of the Refampcin resistant Mycobacterium tuberculosis from study subjects.

\subsection{Sputum Collection and Processing of Sputum Samples for Microscopic Examination}

Three sputum specimens were collected from every consenting TB suspect using coded and sterile plastic containers by laboratory personnel according to national TLCP guideline (Ministry of Health of Ethiopia (MOH) [6]. Portion of the collected specimen was used for direct smear microscopy immediately and the remaining portion was stored at $40 \mathrm{C}$ in refrigerator until further molecular analysis done. Purulent portion of sputum sample was taken and smear was made on one end frosted slide. After air dry, the smear was stained using standard procedure and examined under100X objective of bright field Microscope. The stored portions of three sputum samples of each study subject were pooled in one container for GenXpert analysis.

\subsection{Processing of Sputum Samples for Gene Xpert}

$1 \mathrm{ml}$ of pooled sputum sample was mixed with $2 \mathrm{ml}$ of buffer (Cepheid AB Rontgenvagen 5 SE-171 54, Solna) to liquefy the sputum and incubated at room temperature for 10 minutes. Thereafter, $2 \mathrm{ml}$ of the diluted sample was transferred to the cartridge (Cepheid AB Rontgenvagen 5 SE17154 , Solna) for ultrasonic lysis of mycobacteria to release target DNA. The cartridge was loaded into the Gene Xpert machine (Cepheid) to proceed with the rest protocol. After 2 hours, the comprehensive test result was read on computer screen [11].

\subsection{Ethical Clearance}

Institutional ethical clearance was obtained from the Institutional Review Committee of Hawassa University Medicine and Health Sciences College. Moreover official permission was obtained from Yirgalem Hospital. In addition, the consent was obtained voluntary study subject. All the information obtained from study subject was kept confidential.

\subsection{Statistical Analysis}

Data will be cleaned, edited, coded after it was entered into 
Epi Info version 3.5.1 and exported to SPSS version 16. Using SPSS version 16, descriptive statistics will be used to determine indices. For categorical data, we used proportions with $95 \%$ confidence intervals, Odds ratio and Chi-square test to compare different groups. P values of less than 0.05 were considered statistically significant.

\section{Result}

A total of 236 participants were included under this study. Among these, males (57.6\%) are slightly dominating female. The mean age of the study subjects was $36.9 \pm 13.6$ with the minimum and maximum ages were 2 and 80 years old respectively. Regarding to HIV status, eleven $(4.7 \%)$ of the study subjects were HIV positive and the remaining 146 $(61.9 \%)$ and $79(33.5 \%)$ are negative and unknown status respectively. Concerning to treatment history, $177(75.0 \%)$ are new and the rest 59 (25.0\%) retreated. Fifty eight (24.6\%) of the total subjects were suspected for MDR tuberculosis. Twenty two $(9.3 \%)$ of the subjects were smear positive and thirty nine (16.5\%) Mtb positive under Xpert MTB/RIF test. Among positive sample, eight $(3.4 \%)$ were contained Rifampcin resistant.

The overall prevalence of pulmonary tuberculosis was $16.5 \%$. The disease was slightly predominating among male study subjects. From this prevalence, $3.4 \%$ was shared by Rifampcin mono-resistant Tuberculosis. Based on finding depicted on Table 1, the overall prevalence of Rifampicin Mono-Resistance Mycobacterium tuberculosis among study subject was 3.4\%. Based on Xpert MTB/RIF assay, highest positive finding of rifampcin susceptible Mycobacterium tuberculosis bacilli observed within age group of 16-30. Similarly, rifampcin resistant Mycobacterium tuberculosis detected among productive age group. Based on Fisher's exact test, the positive test result strongly associated with age of the study subjects $(p<0.001)$. Furthermore the microscopic result strongly associated with Xpert MTB/RIF test result $(p<0.001)$. On the other hand the sex of the study subjects has no association with the positive finding $(p>0.05)$.

Table 1. The distribution Rifampcin mono resistant Mycobacterium tuberculosis of the finding among age group of study subjectsfrom AugustDecember, 2014 at Yirgalem Hospital.

\begin{tabular}{|c|c|c|c|c|c|}
\hline \multirow{2}{*}{$\begin{array}{l}\text { Age } \\
\text { group }\end{array}$} & \multicolumn{3}{|c|}{ result of $\mathrm{MTB} / \mathrm{rif}$} & \multirow{2}{*}{ Total } & \multirow{2}{*}{$\begin{array}{l}P \\
\text { Value }\end{array}$} \\
\hline & MTB+/Rif- & MTB+/Rif+ & MTB-/Rif- & & \\
\hline $1-15$ & $4(44.4 \%)$ & $1(11.1 \%)$ & $4(44.4 \%)$ & $9(100.0 \%)$ & \\
\hline $16-30$ & $18(22.8 \%)$ & $3(3.8 \%)$ & $58(73.4 \%)$ & $79(100.0 \%)$ & \\
\hline $31-45$ & $2(2.2 \%)$ & $4(4.3 \%)$ & $86(93.5 \%)$ & $92(100.0 \%)$ & \\
\hline $46-60$ & $7(14.9 \%)$ & $0(0.0 \%)$ & $40(85.1 \%)$ & $47(100.0 \%)$ & \\
\hline $61+$ & $0(0.0 \%)$ & $0(0.0 \%)$ & $9(100.0 \%)$ & $9(100.0 \%)$ & \\
\hline Total & $31(13.1 \%)$ & $8(3.4 \%)$ & $197(83.5 \%)$ & $236(100.0 \%)$ & \\
\hline
\end{tabular}

*Based on Fisher's exact test
Table 2. The distribution Rifampcin mono resistant Mycobacterium tuberculosis of the finding among HIV status of study subjects from AugustDecember, 2014 at Yirgalem Hospital.

\begin{tabular}{lllll}
\hline \multirow{2}{*}{ HIV status } & \multicolumn{2}{l}{ result of MTB/rif result } & \multicolumn{2}{l}{ Total } \\
\cline { 2 - 4 } & MTB+/Rif- & MTB+/Rif+ & MTB-/Rif- & \\
\hline Positive & $2(0.8 \%)$ & $1(0.4 \%)$ & $8(3.4 \%)$ & $11(4.7 \%)$ \\
Negative & $16(6.8 \%)$ & $2(0.8 \%)$ & $\begin{array}{l}128 \\
(54.2 \%)\end{array}$ & $\begin{array}{l}146 \\
(61.9 \%)\end{array}$ \\
Unknown & $13(5.5 \%)$ & $5(2.1 \%)$ & $61(25.8 \%)$ & $\begin{array}{l}79 \\
(33.5 \%)\end{array}$ \\
Total & $31(13.1 \%)$ & $8(3.4 \%)$ & $\begin{array}{l}197 \\
(83.5 \%)\end{array}$ & $\begin{array}{l}236 \\
(100 \%)\end{array}$ \\
\hline
\end{tabular}

\section{Discussion}

Multidrug-resistant (MDR) TB has become a major public health problem and presents new barriers to the control of TB It is due to human error as the result of poor supply management and quality of anti-TB drugs and inadequate or improper treatment, which is further exacerbated by human immunodeficiency virus (HIV) [12].Based on WHO recommendation, RMPMR was considered as surrogate marker for MDR TB. Therefore, we have determined the prevalence of Refampcin mono resistant Mycobacterium tuberculosis among suspected study subject and the finding associated with age and sex.

The overall prevalence of Rifampicin Mono-Resistance Mycobacterium tuberculosis among study subject was 3.4\%. The finding of the current studya higher than study conducted in northern and Eastern part of Ethiopia with prevalence of $2.5 \%$ and $1.7 \%[13,14]$. This may possibly due to unorganized patient diagnosis, treatment, and follow-up and poor patient adherence that may contribute to the higher prevalence of REF monoresistance strains in the study area. On the other hand our finding is lower than similar study conducted in Nigeria (18.8\%) and South Africa 4.9\%) than our study $[15,16]$. This may reflect the variations in sample size, studied population, access to health care facilities, geography and effectiveness of TB control programs.

Based on the study finding, the prevalence of pulmonary Tuberculosis in study area is $16.5 \%$. The study conducted on prevalence of pulmonary tuberculosis in southern Ethiopia showed that higher (19.4\%) than current study [17]. On the other study conducted in south western part of Ethiopia shown to be lower than our finding [18]. The probable reason for this variation could be time of study, difference in study population, method of study and difference in study setting. According to our study the disease was slightly predominating among male study subjects. This is comparable with study conducted in northern Ethiopia [19]

Based on current study result pulmonary tuberculoses was more prevalent among productive age group of the study participants $(11.4 \%)$.This is in agreement with study conducted in Agaro teaching health center in south western Ethiopia [18]. 


\section{Conclusion}

Based on our study finding, The overall prevalence of pulmonary tuberculosis was $16.5 \%$. From these, $3.4 \%$ was account for Rifampicin Mono-Resistance Mycobacterium tuberculosis among study subject. Most of the affected study subjects were productive age group. Therefore, we recommend that there should be enhanced efforts in detection of MDR tuberculosis in study area to control dissemination of the disease among the community.

\section{Acknowledgements}

We would like to appreciate our study subject for their patience to give us the necessary information for answering the research questions.

\section{Authors' Contributions}

Mesfin Worku conceived and designed the study, performed analysis, Interpretation of data, and drafts the manuscript. Teshome Befikadu performed Laboratory Diagnosis and assisted with the design, performed analysis, interpretation of data and the critical review of the manuscript. Both authors read and approved the final manuscript. Both authors participated in critical appraisal and revision of the manuscript.

\section{References}

[1] Murray, C.J. (2000). A Critical Examination of Summary Measures of Population Health. Buletin. WHO 78, 981-994.

[2] Jean D. Wilson et al Harrison's Principles of Internal Medicine. Thomas A. Daniel. Tuberculosis 12th Ed. 1991.

[3] World Health Organisation (WHO): Global tuberculosis control: Surveillance, planning and financing. WHO report. WHO/HTM TB/362. Genev2006.

[4] World Health Organization. WHO Report 2005. Global tuberculosis control: surveillance, planning and financing. Geneva, Switzerland: WHO, 2005.

[5] World Health Organisation (WHO): Globaltuberculosis control: Surveillance, planning and financing. WHO report. WHO/HTM/TB/2008.393. Geneva 2008.

[6] Ministry of Health of Ethiopia (MOH): Tuberculosis, Leprosy and TB/HIV Prevention and Control Programme Manual. Addis Ababa: $M O H 4$ th edition. 2008.

[7] World Health Organization. Assessing tuberculosis prevalence through population-basedsurveys. Geneva: World Health Organization; 2007
[8] Southern Ethiopia Regional State Health Bureau: The Health Sector Development Plan (2000-2004). Awassa, RHB; 2001.

[9] Lindtjørn B, Madebo T: The outcome of tuberculosis treatment at a rural hospital in Southern Ethiopia. Tropical Doctor 2001, 31:132-135.

[10] Yassin MA, Takele L, Gebresenbet S, Girma E, Lera M, Lendebo E, Cuevas LE: HIV and Tuberculosis Coinfection in the SouthernRegion of Ethiopia: A Prospective Epidemiological Study. Scand J Infect Dis 2004, 36:670-673.

[11] Bello LA, Shittu MO, Shittu BT, Oluremi AS, Akinnuroju ON, Adekola SA. Rifampicin-monoresistant Mycobacterium tuberculosis among the patients visiting chest clinic, state specialist hospital, Akure, Nigeria. Int J Res Med Sci 2014; 2:1134-7.

[12] BiadglegneF., Sack U., RodloffAC. Multidrug-resistant tuberculosis in Ethiopia: efforts to expand diagnostic services, treatment and care, journal of antimicrobial resistance and infection control, http://www.aricjournal.com/content/3/1/31

[13] Nigus DM, Lingerew WM, Beyene BA, Tamiru AA, Lemma MT, et al. (2014) Prevalence of Multi Drug Resistant Tuberculosis among Presumptive Multi Drug Resistant Tuberculosis Cases in Amhara National Regional State, Ethiopia. J Mycobac Dis 4: 152.

[14] Seyoum B., Demissie M, Worku A, Bekele S, Aseffa A. Prevalence and Drug Resistance Patterns of Mycobacterium tuberculosis among New Smear Positive Pulmonary Tuberculosis Patients in Eastern Ethiopia, Tuberculosis Research and Treatment, http://dx.doi.org/10.1155/2014/753492

[15] BelloLA,. Shittu MO,. Shittu BT, Oluremi AS,. Akinnuroju ON, Adekola SA. Rifampicinmonoresistant Mycobacterium tuberculosis among the patients visiting chest clinic, state specialist hospital, Akure, Nigeria, International Journal of Research in Medical Sciences, 2014, 2 (3): 1134-1137.

[16] Lawn SD, Brooks SV, Kranzer K, Nicol MP, Whitelaw A, et al. (2011) Screening for HIV Associated Tuberculosis and Rifampicin Resistance before Antiretroviral Therapy Using the Xpert MTB/RIF Assay: A Prospective Study. PLoS Med 8(7): e1001067. doi:10.1371/journal.pmed.1001067.

[17] Zerihun Z., Girmay M., Adane W., Gobena A. Prevalence of Pulmonary Tuberculosis and Associated Risk Factors in Prisons of Gamo Goffa Zone, South Ethiopia: A CrossSectional Study. American Journal of Health Research. Vol. 2, No. 5, 2014, pp. 291-297.

[18] Ali H., Zeynudin A., Mekonnen A., Solomon A., Ali S.Smear posetive pulmonary tuberculosis (ptb) prevalence amongst patients at agaro teaching health center, south west Ethiopia, Ethiop J Health Sci, 2012, 22(1):71-76

[19] Berhe G., Enqueselassie F., Hailu E., Mekonnen W., TekluT. Gebretsadik A., Berhe R., Haile T. Aseffa A. Population-based prevalence survey of tuberculosis in the Tigray region of Ethiopia, BMC Infectious Diseases 2013, 13:448. 